\title{
INEQUALITY FOR RICCI CURVATURE OF CERTAIN SUBMANIFOLDS IN LOCALLY CONFORMAL ALMOST COSYMPLECTIC MANIFOLDS
}

\author{
DAE WON YOON
}

Received 23 June 2004

We establish inequalities between the Ricci curvature and the squared mean curvature, and also between the $k$-Ricci curvature and the scalar curvature for a slant, semi-slant, and bi-slant submanifold in a locally conformal almost cosymplectic manifold with arbitrary codimension.

\section{Preliminaries}

Let $\widetilde{M}$ be a $(2 m+1)$-dimensional almost contact manifold with almost contact structure $(\varphi, \xi, \eta)$, that is, a global vector field $\xi$, a $(1,1)$ tensor field $\varphi$, and a 1 -form $\eta$ on $\widetilde{M}$ such that $\varphi^{2} X=-X+\eta(X) \xi, \eta(\xi)=1$ for any vector field $X$ on $\widetilde{M}$. We consider a product manifold $\widetilde{M} \times \mathbb{R}$, where $\mathbb{R}$ denotes a real line. Then a vector field on $\widetilde{M} \times \mathbb{R}$ is given by $(X, f(d / d t))$, where $X$ is a vector field tangent to $\widetilde{M}, t$ the coordinate of $\mathbb{R}$, and $f$ a function on $\widetilde{M} \times$ $\mathbb{R}$. We define a linear map $J$ on the tangent space of $\widetilde{M} \times \mathbb{R}$ by $J(X, f(d / d t))=(\varphi X-$ $f \xi, \eta(X)(d / d t))$. Then we have $J^{2}=-I$, and hence $J$ is an almost complex structure on $\widetilde{M} \times \mathbb{R}$. The manifold $\widetilde{M}$ is said to be normal (see [6]) if the almost complex structure $J$ is integrable (i.e., $J$ arises from a complex structure on $\widetilde{M} \times \mathbb{R}$ ). Let $g$ be a Riemannian metric on $\widetilde{M}$ compatible with $(\varphi, \xi, \eta)$, that is, $g(\varphi X, \varphi Y)=g(X, Y)-\eta(X) \eta(Y)$ for any vector fields $X$ and $Y$ tangent to $\widetilde{M}$. Thus, the manifold $\widetilde{M}$ is almost contact metric, and $(\varphi, \xi, \eta, g)$ is its almost contact metric structure. Clearly, we have $\eta(X)=g(X, \xi)$ for any vector field $X$ tangent to $\widetilde{M}$. Let $\Phi$ denote the fundamental 2-form of $\widetilde{M}$ defined by $\Phi(X, Y)=g(\varphi X, Y)$ for any vector fields $X$ and $Y$ tangent to $\widetilde{M}$. The manifold $\widetilde{M}$ is said to be almost cosymplectic if the forms $\eta$ and $\Phi$ are closed. That is, $d \eta=0$ and $d \Phi=0$, where $d$ is the operator of exterior differentiation. If $\widetilde{M}$ is almost cosymplectic and normal, then it is called cosymplectic (see[1]). It is well known that the almost contact metric manifold is cosymplectic if and only if $\tilde{\nabla} \varphi$ vanishes identically, where $\tilde{\nabla}$ is the Levi-Civita connection on $\widetilde{M}$. An almost contact metric manifold $\widetilde{M}$ is a locally conformal almost cosymplectic manifold if and only if there exists a 1 -form $\omega$ such that $d \Phi=2 \omega \wedge \Phi, d \eta=\omega \wedge \eta$, and $d \omega=0$.

On the other hand, it is wellknown that the Riemannian curvature tensor $\tilde{R}$ on a locally conformal almost cosymplectic manifold $\widetilde{M}(m \geq 2)$ of pointwise constant $\varphi$-sectional 
curvature $c$ satisfies (see[6])

$$
\begin{aligned}
g(\tilde{R}(X, Y) Z, W) & \\
= & \frac{c-3 f^{2}}{4}\{g(X, W) g(Y, Z)-g(X, Z) g(Y, W)\} \\
& +\frac{c+f^{2}}{4}\{g(X, \varphi W) g(Y, \varphi Z)-g(X, \varphi Z) g(Y, \varphi W)-2 g(X, \varphi Y) g(Z, \varphi W)\} \\
& -\left(\frac{c+f^{2}}{4}+f^{\prime}\right)\{g(X, W) \eta(Y) \eta(Z)-g(X, Z) \eta(Y) \eta(W)+g(Y, Z) \eta(X) \eta(W) \\
& -g(Y, W) \eta(X) \eta(Z)\}, \quad X, Y, Z, W \in T_{p} M,
\end{aligned}
$$

where $f$ is the function such that $\omega=f \eta, f^{\prime}=\xi f$.

In [5], Lotta has introduced the following notion of slant submanifolds into almost contact metric manifolds. A submanifold $M$ tangent to $\xi$ in locally conformal almost cosymplectic manifold $\widetilde{M}$ is said to be slant if for any $p \in M$ and any $X \in T_{p} M$, linearly independent of $\xi$, the angle between $\varphi X$ and $T_{p} M$ is a constant $\theta \in[0, \pi / 2]$, called the slant angle of $M$ in $\widetilde{M}$. Invariant and anti-invariant submanifolds of $\widetilde{M}$ are slant submanifolds with slant angles $\theta=0$ and $\theta=\pi / 2$, respectively.

We say that a submanifold $M$ tangent to $\xi$ is a bi-slant submanifold in $\widetilde{M}$ if there exist two orthogonal distributions $\mathscr{D}_{1}$ and $\mathscr{D}_{2}$ on $M$ such that

(1) $T M$ admits the orthogonal direct decomposition $T M=\mathscr{D}_{1} \oplus \mathscr{D}_{2} \oplus\{\xi\}$;

(2) for any $i=1,2, \mathscr{D}_{i}$ is slant distribution with slant angle $\theta_{i}$.

On the other hand, $C R$-submanifolds of $\widetilde{M}$ are bi-slant submanifolds with $\theta_{1}=0, \theta_{2}=$ $\pi / 2$.

Let $2 d_{1}=\operatorname{dim} \mathscr{D}_{1}$ and $2 d_{2}=\operatorname{dim} \mathscr{D}_{2}$.

Remark 1.1. If either $d_{1}$ or $d_{2}$ vanishes, the bi-slant submanifold is a slant submanifold. Thus, slant submanifolds are particular cases of bi-slant submanifolds.

A submanifold $M$ tangent to $\xi$ is called a semi-slant submanifold in $\widetilde{M}$ if there exist two orthogonal distributions $\mathscr{D}_{1}$ and $\mathscr{D}_{2}$ on $M$ such that

(1) $T M$ admits the orthogonal direct decomposition $T M=\mathscr{D}_{1} \oplus \mathscr{D}_{2} \oplus\{\xi\}$;

(2) the distribution $\mathscr{D}_{1}$ is an invariant distribution, that is, $\varphi\left(\mathscr{D}_{1}\right)=\mathscr{D}_{1}$;

(3) the distribution $\mathscr{D}_{2}$ is slant with angle $\theta \neq 0$.

Remark 1.2. The invariant distribution of a semi-slant submanifold is a slant distribution with zero angle. Thus, it is obvious that, in fact, semi-slant submanifolds are particular cases of bi-slant submanifolds.

(1) If $d_{2}=0$, then $M$ is an invariant submanifold.

(2) If $d_{1}=0$ and $\theta=\pi / 2$, then $M$ is an anti-invariant submanifold.

For the other properties and examples of slant, bi-slant, and semi-slant submanifolds in an almost contact metric manifold, we refer to $[2,3]$.

Let $M$ be an $n$-dimensional submanifold of a locally conformal almost cosymplectic manifold $\widetilde{M}$ equipped with a Riemannian metric $g$. The Gauss and Weingarten formulas 
are given, respectively, by

$$
\tilde{\nabla}_{X} Y=\nabla_{X} Y+h(X, Y), \quad \tilde{\nabla}_{X} N=-A_{N} X+\nabla_{X}^{\perp} N
$$

for all $X, Y \in T M$ and $N \in T^{\perp} M$, where $\tilde{\nabla}, \nabla$, and $\nabla^{\perp}$ are the Riemannian, induced Riemannian, and induced normal connections in $\widetilde{M}, M$, and the normal bundle $T^{\perp} M$ of $M$, respectively, and $h$ is the second fundamental form related to the shape operator $A$ by $g(h(X, Y), N)=g\left(A_{N} X, Y\right)$. Also, let $R$ be the Riemannian curvature tensor of $M$. Then the equation of Gauss is given by

$$
\tilde{R}(X, Y, Z, W)=R(X, Y, Z, W)+g(h(X, W), h(Y, Z))-g(h(X, Z), h(Y, W)),
$$

for any vectors $X, Y, Z, W$ tangent to $M$.

For any vector $X$ tangent to $M$, we put $\varphi X=P X+F X$, where $P X$ and $F X$ are the tangential and the normal components of $\varphi X$, respectively. Given an orthonormal basis $\left\{e_{1}, \ldots, e_{n}\right\}$ of $M$, we define the squared norm of $P$ by

$$
\|P\|^{2}=\sum_{i, j=1}^{n} g^{2}\left(P e_{i}, e_{j}\right)
$$

and the mean curvature vector $H(p)$ at $p \in M$ is given by $H=(1 / n) \sum_{i=1}^{n} h\left(e_{i}, e_{i}\right)$.

We put

$$
h_{i j}^{r}=g\left(h\left(e_{i}, e_{j}\right), e_{r}\right), \quad\|h\|^{2}=\sum_{i, j=1}^{n} g\left(h\left(e_{i}, e_{j}\right), h\left(e_{i}, e_{j}\right)\right),
$$

where $\left\{e_{n+1}, \ldots, e_{2 m+1}\right\}$ is an orthonormal basis of $T_{p}^{\perp} M$ and $r=n+1, \ldots, 2 m+1$. A submanifold $M$ in $\widetilde{M}$ is called totally geodesic if the second fundamental form vanishes identically and totally umbilical if there is a real number $\lambda$ such that $h(X, Y)=\lambda g(X, Y) H$ for any tangent vectors $X, Y$ on $M$.

For an $n$-dimensional Riemannian manifold $M$, we denote by $K(\pi)$ the sectional curvature of $M$ associated with a plane section $\pi \subset T_{p} M, p \in M$. For an orthonormal basis $\left\{e_{1}, \ldots, e_{n}\right\}$ of the tangent space $T_{p} M$, the scalar curvature $\tau$ is defined by

$$
\tau=\sum_{i<j} K_{i j}
$$

where $K_{i j}$ denotes the sectional curvature of the 2-plane section spanned by $e_{i}$ and $e_{j}$.

Suppose that $L$ is a $k$-plane section of $T_{p} M$ and $X$ a unit vector in $L$. We choose an orthonormal basis $\left\{e_{1}, \ldots, e_{k}\right\}$ of $L$ such that $e_{1}=X$. Define the Ricci curvature $\operatorname{Ric}_{L}$ of $L$ at $X$ by

$$
\operatorname{Ric}_{L}(X)=K_{12}+\cdots+K_{1 k}
$$




\section{Inequality for Ricci curvature}

We simply called such a curvature a $k$-Ricci curvature. The scalar curvature $\tau$ of the $k$ plane section $L$ is given by

$$
\tau(L)=\sum_{1 \leq i<j \leq k} K_{i j}
$$

For each integer $k, 2 \leq k \leq n$, the Riemannain invariant $\Theta_{k}$ on an $n$-dimensional Riemannian manifold $M$ is defined by

$$
\Theta_{k}(p)=\frac{1}{k-1} \inf _{L, X} \operatorname{Ric}_{L}(X), \quad p \in M,
$$

where $L$ runs over all $k$-plane sections in $T_{p} M$ and $X$ runs over all unit vectors in $L$.

Recall that for a submanifold $M$ in a Riemannain manifold, the relative null space of $M$ at a point $p \in M$ is defined by

$$
N_{p}=\left\{X \in T_{p} M \mid h(X, Y)=0 \forall Y \in T_{p} M\right\}
$$

\section{Ricci curvature and squared mean curvature}

Chen established a sharp relationship between the Ricci curvature and the squared mean curvature for submanifolds in real space forms (see [4]). We prove similar inequalities for slant, bi-slant, and semi-slant submanifolds in a locally conformal almost cosymplectic manifold $\widetilde{M}$. We consider submanifolds $M$ tangent to $\xi$.

Theorem 2.1. Let $M$ be an $n$-dimensional $\theta$-slant submanifold tangent to $\xi$ into a $(2 m+1)$ dimensional locally conformal almost cosymplectic manifold $\widetilde{M}$. Then, the following hold.

(1) For each unit vector $X \in T_{p} M$ orthogonal to $\xi$,

$$
\operatorname{Ric}(X) \leq \frac{1}{4}\left\{(n-1)\left(c-3 f^{2}\right)+\frac{3}{2}\left(c+f^{2}\right) \cos ^{2} \theta-4\left(\frac{c+f^{2}}{4}+f^{\prime}\right)+n^{2}\|H\|^{2}\right\} .
$$

(2) If $H(p)=0$, then a unit tangent vector $X$ orthogonal to $\xi$ at $p$ satisfies the equality case of (2.1) if and only if $X \in N_{p}$.

(3) The equality case of (2.1) holds identically for all unit tangent vectors orthogonal to $\xi$ at $p$ if and only if $p$ is a totally geodesic point.

Proof. (1) Let $X \in T_{p} M$ be a unit tangent vector at $p$ orthogonal to $\xi$. We choose an orthonormal basis $e_{1}, \ldots, e_{n}=\xi, e_{n+1}, \ldots, e_{2 m+1}$, such that $e_{1}, \ldots, e_{n}$ are tangent to $M$ at $p$ with $e_{1}=X$. Then, from the equation of Gauss, we have

$$
\begin{aligned}
n^{2}\|H\|^{2}= & 2 \tau+\|h\|^{2}-\frac{n(n-1)\left(c-3 f^{2}\right)}{4} \\
& -\frac{3(n-1)\left(c+f^{2}\right)}{4} \cos ^{2} \theta+2(n-1)\left(\frac{c+f^{2}}{4}+f^{\prime}\right) .
\end{aligned}
$$


From (2.2), we get

$$
\begin{aligned}
n^{2}\|H\|^{2}= & 2 \tau+\sum_{r=n+1}^{2 m+1}\left[\left(h_{11}^{r}\right)^{2}+\left(h_{22}^{r}+\cdots+h_{n n}^{r}\right)^{2}+2 \sum_{1 \leq i<j \leq n}\left(h_{i j}^{r}\right)^{2}\right] \\
& -2 \sum_{r=n+1}^{2 m+1} \sum_{2 \leq i<j \leq n} h_{i i}^{r} h_{j j}^{r}-\frac{n(n-1)\left(c-3 f^{2}\right)}{4} \\
& -\frac{3(n-1)\left(c+f^{2}\right)}{4} \cos ^{2} \theta+2(n-1)\left(\frac{c+f^{2}}{4}+f^{\prime}\right) \\
= & 2 \tau+\frac{1}{2} \sum_{r=n+1}^{2 m+1}\left[\left(h_{11}^{r}+h_{22}^{r}+\cdots+h_{n n}^{r}\right)^{2}+\left(h_{11}^{r}-h_{22}^{r}-\cdots-h_{n n}^{r}\right)^{2}\right] \\
& +2 \sum_{r=n+1}^{2 m+1} \sum_{1 \leq i<j \leq n}\left(h_{i j}^{r}\right)^{2}-2 \sum_{r=n+1}^{2 m+1} \sum_{i<i \leq n} h_{i i}^{r} h_{j j}^{r} \\
& -\frac{n(n-1)\left(c-3 f^{2}\right)}{4}-\frac{3(n-1)\left(c+f^{2}\right)}{4} \cos ^{2} \theta+2(n-1)\left(\frac{c+f^{2}}{4}+f^{\prime}\right) .
\end{aligned}
$$

By using the equation of Gauss, we have

$$
\begin{aligned}
\sum_{2 \leq i<j \leq n} K_{i j}= & \sum_{r=n+1}^{2 m+1} \sum_{2 \leq i<j \leq n}\left[h_{i i}^{r} h_{j j}^{r}-\left(h_{i j}^{r}\right)^{2}\right]+\frac{(n-1)(n-2)\left(c-3 f^{2}\right)}{8} \\
& +\frac{3(n-2)\left(c+f^{2}\right)}{8} \cos ^{2} \theta+\frac{1}{2}\left(\frac{c+f^{2}}{4}+f^{\prime}\right)(-2 n+4) .
\end{aligned}
$$

Substituting (2.4) in (2.3), we get

$$
\frac{1}{2} n^{2}\|H\|^{2} \geq 2 \operatorname{Ric}(X)-\frac{(n-1)\left(c-3 f^{2}\right)}{2}-\frac{3\left(c+f^{2}\right)}{4} \cos ^{2} \theta+2\left(\frac{c+f^{2}}{4}+f^{\prime}\right)
$$

or equivalently (2.1).

(2) Assume that $H(P)=0$. Equality holds in (2.1) if and only if

$$
\begin{gathered}
h_{12}^{r}=\cdots=h_{1 n}^{r}=0, \\
h_{11}^{r}=h_{22}^{r}+\cdots+h_{n n}^{r}, \quad r \in\{n+1, \ldots, 2 m+1\} .
\end{gathered}
$$

Then $h_{1 j}^{r}=0$ for all $j \in\{1, \ldots, n\}, r \in\{n+1, \ldots, 2 m+1\}$, that is, $X \in N_{p}$.

(3) Then equality case of (2.1) holds for all unit tangent vectors orthogonal to $\xi$ at $p$ if and only if

$$
\begin{gathered}
h_{i j}^{r}=0, \quad i \neq j, r \in\{n+1, \ldots, 2 m+1\}, \\
h_{11}^{r}+\cdots+h_{n n}^{r}-2 h_{i i}^{r}=0, \quad i \in\{1, \ldots, n\}, r \in\{n+1, \ldots, 2 m+1\} .
\end{gathered}
$$

In this case, it follows that $p$ is a totally geodesic point. The converse is trivial. 
Theorem 2.2. Let $M$ be an n-dimensional bi-slant submanifold satisfying $g(X, \varphi Y)=0$, for any $X \in \mathscr{D}_{1}$ and any $Y \in \mathscr{D}_{2}$, tangent to $\xi$ in a $(2 m+1)$-dimensional locally conformal almost cosymplectic manifold $\widetilde{M}$. Then, the following hold.

(1) For each unit vector $X \in T_{p} M$ orthogonal to $\xi$ and if

(i) $X$ is tangent to $\mathscr{D}_{1}$,

$$
\operatorname{Ric}(X) \leq \frac{1}{4}\left\{(n-1)\left(c-3 f^{2}\right)+\frac{3}{2}\left(c+f^{2}\right) \cos ^{2} \theta_{1}-4\left(\frac{c+f^{2}}{4}+f^{\prime}\right)+n^{2}\|H\|^{2}\right\}
$$

and if

(ii) $X$ is tangent to $\mathscr{D}_{2}$,

$$
\operatorname{Ric}(X) \leq \frac{1}{4}\left\{(n-1)\left(c-3 f^{2}\right)+\frac{3}{2}\left(c+f^{2}\right) \cos ^{2} \theta_{2}-4\left(\frac{c+f^{2}}{4}+f^{\prime}\right)+n^{2}\|H\|^{2}\right\}
$$

(2) If $H(p)=0$, then a unit tangent vector $X$ orthogonal to $\xi$ at $p$ satisfies the equality case of (2.8) and (2.9) if and only if $X \in N_{p}$.

(3) The equality case of (2.8) and (2.9) holds identically for all unit tangent vectors orthogonal to $\xi$ at $p$ if and only if $p$ is a totally geodesic point.

Proof. (1) Let $X \in T_{p} M$ be a unit tangent vector at $p$ orthogonal to $\xi$. We choose an othonormal basis $e_{1}, \ldots, e_{n}=\xi, e_{n+1}, \ldots, e_{2 m+1}$ such that $e_{1}, \ldots, e_{n}$ are tangent to $M$ at $p$ with $e_{1}=X$. Then, from the equation of Gauss, we have

$$
\begin{aligned}
n^{2}\|H\|^{2}= & 2 \tau+\|h\|^{2}-\frac{n(n-1)\left(c-3 f^{2}\right)}{4} \\
& -\frac{6\left(c+f^{2}\right)}{4}\left(d_{1} \cos ^{2} \theta_{1}+d_{2} \cos ^{2} \theta_{2}\right)+2(n-1)\left(\frac{c+f^{2}}{4}+f^{\prime}\right),
\end{aligned}
$$

where $2 d_{1}=\operatorname{dim} \mathscr{D}_{1}$ and $2 d_{2}=\operatorname{dim} \mathscr{D}_{2}$.

From (2.10), we get

$$
\begin{aligned}
n^{2}\|H\|^{2}= & 2 \tau+\sum_{r=n+1}^{2 m+1}\left[\left(h_{11}^{r}\right)^{2}+\left(h_{22}^{r}+\cdots+h_{n n}^{r}\right)^{2}+2 \sum_{1 \leq i<j \leq n}\left(h_{i j}^{r}\right)^{2}\right] \\
& -2 \sum_{r=n+1}^{2 m+1} \sum_{2 \leq i<j \leq n} h_{i i}^{r} h_{j j}^{r}-\frac{n(n-1)\left(c-3 f^{2}\right)}{4} \\
& -\frac{6\left(c+f^{2}\right)}{4}\left(d_{1} \cos ^{2} \theta_{1}+d_{2} \cos ^{2} \theta_{2}\right)+2(n-1)\left(\frac{c+f^{2}}{4}+f^{\prime}\right)
\end{aligned}
$$




$$
\begin{aligned}
= & 2 \tau+\frac{1}{2} \sum_{r=n+1}^{2 m+1}\left[\left(h_{11}^{r}+h_{22}^{r}+\cdots+h_{n n}^{r}\right)^{2}+\left(h_{11}^{r}-h_{22}^{r}-\cdots-h_{n n}^{r}\right)^{2}\right] \\
& +2 \sum_{r=n+1}^{2 m+1} \sum_{1 \leq i<j \leq n}\left(h_{i j}^{r}\right)^{2}-2 \sum_{r=n+1}^{2 m+1} \sum_{2 \leq i<j \leq n} h_{i i}^{r} h_{j j}^{r}-\frac{n(n-1)\left(c-3 f^{2}\right)}{4} \\
& -\frac{6\left(c+f^{2}\right)}{4}\left(d_{1} \cos ^{2} \theta_{1}+d_{2} \cos ^{2} \theta_{2}\right)+2(n-1)\left(\frac{c+f^{2}}{4}+f^{\prime}\right) .
\end{aligned}
$$

We distinguish two cases.

(i) If $X$ is tangent to $\mathscr{D}_{1}$, then we have

$$
\begin{aligned}
\sum_{2 \leq i<j \leq n} K_{i j}= & \sum_{r=n+1}^{2 m+1} \sum_{2 \leq i<j \leq n}\left[h_{i i}^{r} h_{j j}^{r}-\left(h_{i j}^{r}\right)^{2}\right]+\frac{(n-1)(n-2)\left(c-3 f^{2}\right)}{8} \\
& +\frac{c+f^{2}}{8}\left[6\left(d_{1} \cos ^{2} \theta_{1}+d_{2} \cos ^{2} \theta_{2}\right)-3 \cos ^{2} \theta_{1}\right]+\frac{1}{2}\left(\frac{c+f^{2}}{4}+f^{\prime}\right)(-2 n+4) .
\end{aligned}
$$

Substituting (2.12) in (2.11), one gets

$$
\frac{1}{2} n^{2}\|H\|^{2} \geq 2 \operatorname{Ric}(X)-\frac{(n-1)\left(c-3 f^{2}\right)}{2}-\frac{3\left(c+f^{2}\right)}{4} \cos ^{2} \theta_{1}+2\left(\frac{c+f^{2}}{4}+f^{\prime}\right),
$$

which is equivalent to (2.8).

(ii) If $X$ is tangent to $\mathscr{D}_{2}$, then we have

$$
\begin{aligned}
\sum_{2 \leq i<j \leq n} K_{i j}= & \sum_{r=n+1}^{2 m+1} \sum_{2 \leq i<j \leq n}\left[h_{i i}^{r} h_{j j}^{r}-\left(h_{i j}^{r}\right)^{2}\right]+\frac{(n-1)(n-2)\left(c-3 f^{2}\right)}{8} \\
& +\frac{c+f^{2}}{8}\left[6\left(d_{1} \cos ^{2} \theta_{1}+d_{2} \cos ^{2} \theta_{2}\right)-3 \cos ^{2} \theta_{2}\right]+\frac{1}{2}\left(\frac{c+f^{2}}{4}+f^{\prime}\right)(-2 n+4) .
\end{aligned}
$$

Substituting (2.14) in (2.11), one gets

$$
\frac{1}{2} n^{2}\|H\|^{2} \geq 2 \operatorname{Ric}(X)-\frac{(n-1)\left(c-3 f^{2}\right)}{2}-\frac{3\left(c+f^{2}\right)}{4} \cos ^{2} \theta_{2}+2\left(\frac{c+f^{2}}{4}+f^{\prime}\right),
$$

which is equivalent to (2.9).

(2) Assume that $H(p)=0$. Equality holds in (2.8) and (2.9) if and only if

$$
\begin{gathered}
h_{12}^{r}=\cdots=h_{1 n}^{r}=0, \\
h_{11}^{r}=h_{22}^{r}+\cdots+h_{n n}^{r}, \quad r \in\{n+1, \ldots, 2 m+1\} .
\end{gathered}
$$

Then $h_{1 j}^{r}=0$ for all $j \in\{1, \ldots, n\}, r \in\{n+1, \ldots, 2 m+1\}$, that is, $X \in N_{p}$. 
(3) Then equality case of (2.8) and (2.9) holds for all unit tangent vectors orthogonal to $\xi$ at $p$ if and only if

$$
\begin{gathered}
h_{i j}^{r}=0, \quad i \neq j, r \in\{n+1, \ldots, 2 m+1\}, \\
h_{11}^{r}+\cdots+h_{n n}^{r}-2 h_{i i}^{r}=0, \quad i \in\{1, \ldots, n\}, r \in\{n+1, \ldots, 2 m+1\} .
\end{gathered}
$$

In this case, it follows that $p$ is a totally geodesic point. The converse is trivial.

Corollary 2.3. Let $M$ be an $n$-dimensional semi-slant submanifold in a $(2 m+1)$ dimensional locally conformal almost cosymplectic manifold $\widetilde{M}$. Then, the following hold.

(1) For each unit vector $X \in T_{p} M$ orthogonal to $\xi$ and if

(i) $X$ is tangent to $\mathscr{D}_{1}$,

$$
\operatorname{Ric}(X) \leq \frac{1}{4}\left\{(n-1)\left(c-3 f^{2}\right)-4\left(\frac{c+f^{2}}{4}+f^{\prime}\right)+n^{2}\|H\|^{2}\right\},
$$

and if

(ii) $X$ is tangent to $\mathscr{D}_{2}$,

$$
\operatorname{Ric}(X) \leq \frac{1}{4}\left\{(n-1)\left(c-3 f^{2}\right)+\frac{3}{2}\left(c+f^{2}\right) \cos ^{2} \theta-4\left(\frac{c+f^{2}}{4}+f^{\prime}\right)+n^{2}\|H\|^{2}\right\} .
$$

(2) If $H(p)=0$, then a unit tangent vector $X$ orthogonal to $\xi$ at $p$ satisfies the equality case of (2.18) and (2.19) if and only if $X \in N_{p}$.

(3) The equality case of (2.18) and (2.19) holds identically for all unit tangent vectors orthogonal to $\xi$ at $p$ if and only if $p$ is a totally geodesic point.

Corollary 2.4. Let $M$ be an $n$-dimensional invariant submanifold in a $(2 m+1)$ dimensional cosymplectic space form $\tilde{M}(c)$. Then, the following hold.

(1) For each unit vector $X \in T_{p} M$ orthogonal to $\xi$,

$$
\operatorname{Ric}(X) \leq \frac{1}{4}\left\{(n-1)\left(c-3 f^{2}\right)+\frac{3}{2}\left(c+f^{2}\right)-4\left(\frac{c+f^{2}}{4}+f^{\prime}\right)+n^{2}\|H\|^{2}\right\} .
$$

(2) If $H(p)=0$, then a unit tangent vector $X$ orthogonal to $\xi$ at $p$ satisfies the equality case of (2.20) if and only if $X \in N_{p}$.

(3) The equality case of (2.20) holds identically for all unit tangent vectors orthogonal to $\xi$ at $p$ if and only if $p$ is a totally geodesic point.

Corollary 2.5. Let $M$ be an n-dimensional anti-invariant submanifold in a $(2 m+1)$ dimensional cosymplectic space form $\tilde{M}(c)$. Then, the following hold.

(1) For each unit vector $X \in T_{p} M$ orthogonal to $\xi$,

$$
\operatorname{Ric}(X) \leq \frac{1}{4}\left\{(n-1)\left(c-3 f^{2}\right)-4\left(\frac{c+f^{2}}{4}+f^{\prime}\right)+n^{2}\|H\|^{2}\right\} .
$$

(2) If $H(p)=0$, then a unit tangent vector $X$ orthogonal to $\xi$ at $p$ satisfies the equality case of (2.21) if and only if $X \in N_{p}$. 
(3) The equality case of (2.21) holds identically for all unit tangent vectors orthogonal to $\xi$ at $p$ if and only if $p$ is a totally geodesic point.

\section{3. $k$-Ricci curvature and squared mean curvature}

In this section, we prove relationship between the $k$-Ricci curvature and the squared mean curvature for slant, bi-slant, and semi-slant submanifolds in a locally conformal almost cosymplectic manifold $\widetilde{M}$. We state an inequality between the scalar curvature and the squared mean curvature for submanifolds $M$ tangent to the vector field $\xi$.

Theorem 3.1. Let $M$ be an $n$-dimensional $\theta$-slant submanifold tangent to $\xi$ into a $(2 m+1)$ dimensional locally conformal almost cosymplectic manifold $\widetilde{M}$. Then,

$$
\|H\|^{2} \geq \frac{2 \tau}{n(n-1)}-\frac{1}{4 n}\left[n\left(c-3 f^{2}\right)+3\left(c+f^{2}\right) \cos ^{2} \theta-8\left(\frac{c+f^{2}}{4}+f^{\prime}\right)\right],
$$

equality holding at a point $p \in M$ if and only if $p$ is a totally umbilical point.

Proof. Let $p$ be a point of $M$. We choose an orthonormal basis $\left\{e_{1}, e_{2}, \ldots, e_{n}=\xi\right\}$ for the tangent space $T_{p} M$ and $\left\{e_{n+1}, \ldots, e_{2 m+1}\right\}$ for the normal space $T_{p}^{\perp} M$ at $p$ such that the normal vector $e_{n+1}$ is in the direction of the mean curvature vector and $e_{1}, e_{2}, \ldots, e_{n}$ diagonalize the shape operator $A_{n+1}$. Then, we have

$$
\begin{gathered}
A_{n+1}=\left(\begin{array}{ccccc}
a_{1} & 0 & 0 & \ldots & 0 \\
0 & a_{2} & 0 & \ldots & 0 \\
0 & 0 & a_{3} & \ldots & 0 \\
\vdots & \vdots & \vdots & \ddots & \vdots \\
0 & 0 & 0 & \ldots & a_{n}
\end{array}\right), \\
A_{r}=\left(h_{i j}^{r}\right), \quad \sum_{i=1}^{n} h_{i i}^{r}=0, \quad n+2 \leq r \leq 2 m+1 .
\end{gathered}
$$

From the equation of Gauss,

$$
\begin{aligned}
n^{2}\|H\|^{2}= & 2 \tau+\sum_{i=1}^{n} a_{i}^{2}+\sum_{r=n+2}^{2 m+1} \sum_{i, j=1}^{n}\left(h_{i j}^{r}\right)^{2}-\frac{n(n-1)\left(c-3 f^{2}\right)}{4} \\
& -\frac{3(n-1)\left(c+f^{2}\right)}{4} \cos ^{2} \theta+2(n-1)\left(\frac{c+f^{2}}{4}+f^{\prime}\right) .
\end{aligned}
$$

On the other hand,

$$
\sum_{i<j}\left(a_{i}-a_{j}\right)^{2}=(n-1) \sum_{i=1}^{n} a_{i}^{2}-2 \sum_{i<j} a_{i} a_{j}
$$


1630 Inequality for Ricci curvature

Therefore, from the above equation, we have

$$
n^{2}\|H\|^{2}=\left(\sum_{i=1}^{n} a_{i}\right)^{2}=\sum_{i=1}^{n} a_{i}^{2}+2 \sum_{i<j} a_{i} a_{j} \leq n \sum_{i=1}^{n} a_{i}^{2}
$$

Combining (3.3) and (3.5),

$$
\begin{aligned}
n(n-1)\|H\|^{2} \geq & 2 \tau+\sum_{r=n+2}^{2 m+1} \sum_{i, j=1}^{n}\left(h_{i j}^{r}\right)^{2}-\frac{n(n-1)\left(c-3 f^{2}\right)}{4} \\
& -\frac{3(n-1)\left(c+f^{2}\right)}{4} \cos ^{2} \theta+2(n-1)\left(\frac{c+f^{2}}{4}+f^{\prime}\right),
\end{aligned}
$$

which implies inequality (3.1). If the equality sign of (3.1) holds at a point $p \in M$, then from (3.4) and (3.6) we get $A_{r}=0(r=n+2, \ldots, 2 m+1)$ and $a_{1}=\cdots=a_{n}$. Consequently, $p$ is a totally umbilical point. The converse is trivial.

TheOREM 3.2. Let $M$ be an $n$-dimensional bi-slant submanifold satisfying $g(X, \varphi Y)=0$, for any $X \in \mathscr{D}_{1}$ and any $Y \in \mathscr{D}_{2}$, tangent to $\xi$ into a $(2 m+1)$-dimensional locally conformal almost cosymplectic manifold $\widetilde{M}$. Then,

$$
\begin{aligned}
\|H\|^{2} \geq \frac{2 \tau}{n(n-1)}-\frac{1}{4 n(n-1)}[ & n(n-1)\left(c-3 f^{2}\right)+6\left(d_{1} \cos ^{2} \theta_{1}+d_{2} \cos ^{2} \theta_{2}\right)\left(c+f^{2}\right) \\
& \left.-8(n-1)\left(\frac{c+f^{2}}{4}+f^{\prime}\right)\right],
\end{aligned}
$$

where $2 d_{1}=\operatorname{dim} \mathscr{D}_{1}$ and $2 d_{2}=\operatorname{dim} \mathscr{D}_{2}$.

Theorem 3.3. Let $M$ be an $n$-dimensional semi-slant submanifold tangent to $\xi$ into a $(2 m+$ 1)-dimensional locally conformal almost cosymplectic manifold $\widetilde{M}$. Then,

$$
\begin{aligned}
\|H\|^{2} \geq \frac{2 \tau}{n(n-1)}-\frac{1}{4 n(n-1)}[ & n(n-1)\left(c-3 f^{2}\right)+6\left(d_{1}+d_{2} \cos ^{2} \theta\right)\left(c+f^{2}\right) \\
& \left.-8(n-1)\left(\frac{c+f^{2}}{4}+f^{\prime}\right)\right],
\end{aligned}
$$

where $2 d_{1}=\operatorname{dim} \mathscr{D}_{1}$ and $2 d_{2}=\operatorname{dim} \mathscr{D}_{2}$.

Theorem 3.4. Let $M$ be an $n$-dimensional $\theta$-slant submanifold tangent to $\xi$ into a $(2 m+1)$ dimensional locally conformal almost cosymplectic manifold $\widetilde{M}$. Then, for any integer $k(2 \leq$ $k \leq n)$ and any point $p \in M$,

$$
\|H\|^{2} \geq \Theta_{k}(p)-\frac{1}{4 n}\left[n\left(c-3 f^{2}\right)+3\left(c+f^{2}\right) \cos ^{2} \theta-8\left(\frac{c+f^{2}}{4}+f^{\prime}\right)\right] .
$$


Proof. Let $\left\{e_{1}, \ldots, e_{n}\right\}$ be an orthonormal basis of $T_{p} M$. Denote by $L_{i_{1} \cdots i_{k}}$ the $k$-plane section spanned by $e_{i_{1}}, \ldots, e_{i_{k}}$. It follows from (1.7) and (1.8) that

$$
\begin{aligned}
& \tau\left(L_{i_{1} \cdots i_{k}}\right)=\frac{1}{2} \sum_{i \in\left\{i_{1}, \ldots, i_{k}\right\}} \operatorname{Ric}_{L_{i_{1} \cdots i_{k}}}\left(e_{i}\right), \\
& \tau(p)=\frac{1}{\left(\begin{array}{l}
n-2 \\
k-2
\end{array}\right)} \sum_{1 \leq i_{1}<\cdots<i_{k} \leq n} \tau\left(L_{i_{1} \cdots i_{k}}\right) .
\end{aligned}
$$

Combining (1.9) and (3.10), we obtain

$$
\tau(p) \geq \frac{n(n-1)}{2} \Theta_{k}(p)
$$

Therefore, by using (3.1) and (3.11), we can obtain the inequality in Theorem 3.4.

Theorem 3.5. Let $M$ be an $n$-dimensional bi-slant submanifold tangent to $\xi$ into a $(2 m+$ 1)-dimensional locally conformal almost cosymplectic manifold $\widetilde{M}$. Then, for any integer $k(2 \leq k \leq n)$ and any point $p \in M$,

$$
\begin{aligned}
\|H\|^{2} \geq \Theta_{k}(p)-\frac{1}{4 n(n-1)}[ & n(n-1)\left(c-3 f^{2}\right)+6\left(d_{1} \cos ^{2} \theta_{1}+d_{2} \cos ^{2} \theta_{2}\right)\left(c+f^{2}\right) \\
& \left.-8(n-1)\left(\frac{c+f^{2}}{4}+f^{\prime}\right)\right],
\end{aligned}
$$

where $2 d_{1}=\operatorname{dim} \mathscr{D}_{1}$ and $2 d_{2}=\operatorname{dim} \mathscr{D}_{2}$.

Theorem 3.6. Let $M$ be an $n$-dimensional semi-slant submanifold tangent to $\xi$ into a $(2 m+$ 1)-dimensional locally conformal almost cosymplectic manifold $\widetilde{M}$. Then, for any integer $k(2 \leq k \leq n)$ and any point $p \in M$,

$$
\begin{aligned}
\|H\|^{2} \geq \Theta_{k}(p)-\frac{1}{4 n(n-1)}[ & n(n-1)\left(c-3 f^{2}\right)+6\left(d_{1}+d_{2} \cos ^{2} \theta\right)\left(c+f^{2}\right) \\
& \left.-8(n-1)\left(\frac{c+f^{2}}{4}+f^{\prime}\right)\right],
\end{aligned}
$$

where $2 d_{1}=\operatorname{dim} \mathscr{D}_{1}$ and $2 d_{2}=\operatorname{dim} \mathscr{D}_{2}$.

Corollary 3.7. Let $M$ be an $n$-dimensional invariant submanifold tangent to $\xi$ into a $(2 m+1)$-dimensional locally conformal almost cosymplectic manifold $\widetilde{M}$. Then, for any integer $k(2 \leq k \leq n)$ and any point $p \in M$,

$$
\|H\|^{2} \geq \Theta_{k}(p)-\frac{1}{4 n}\left[n\left(c-3 f^{2}\right)+3\left(c+f^{2}\right)-8\left(\frac{c+f^{2}}{4}+f^{\prime}\right)\right] .
$$




\section{Inequality for Ricci curvature}

Corollary 3.8. Let $M$ be an n-dimensional anti-invariant submanifold tangent to $\xi$ into $a(2 m+1)$-dimensional locally conformal almost cosymplectic manifold $\widetilde{M}$. Then, for any integer $k(2 \leq k \leq n)$ and any point $p \in M$,

$$
\|H\|^{2} \geq \Theta_{k}(p)-\frac{1}{4 n}\left[n\left(c-3 f^{2}\right)-8\left(\frac{c+f^{2}}{4}+f^{\prime}\right)\right] .
$$

Corollary 3.9. Let $M$ be an n-dimensional contact $C R$-submanifold tangent to $\xi$ into $a(2 m+1)$-dimensional locally conformal almost cosymplectic manifold $\widetilde{M}$. Then, for any integer $k(2 \leq k \leq n)$ and any point $p \in M$,

$$
\|H\|^{2} \geq \Theta_{k}(p)-\frac{1}{4 n(n-1)}\left[n(n-1)\left(c-3 f^{2}\right)+6 d_{1}\left(c+f^{2}\right)-8(n-1)\left(\frac{c+f^{2}}{4}+f^{\prime}\right)\right] .
$$

\section{References}

[1] D. E. Blair, Contact Manifolds in Riemannian Geometry, Lecture Notes in Mathematics, vol. 509, Springer, Berlin, 1976.

[2] J. L. Cabrerizo, A. Carriazo, L. M. Fernández, and M. Fernández, Semi-slant submanifolds of a Sasakian manifold, Geom. Dedicata 78 (1999), no. 2, 183-199.

[3] _ Slant submanifolds in Sasakian manifolds, Glasg. Math. J. 42 (2000), no. 1, 125-138.

[4] B.-Y. Chen, Relations between Ricci curvature and shape operator for submanifolds with arbitrary codimensions, Glasg. Math. J. 41 (1999), no. 1, 33-41.

[5] A. Lotta, Slant submanifolds in contact geometry, Bull. Math. Soc. Sci. Math. Roumanie (N.S.) 39 (1996), no. 1-4, 183-198.

[6] Z. Olszak, Locally conformal almost cosymplectic manifolds, Colloq. Math. 57 (1989), no. 1, 7387.

Dae Won Yoon: Department of Mathematics Education and Research Institute of Natural Science (RINS), Gyeongsang National University, 900 Gazwa-dong, Jinju 660-701, South Korea

E-mail address: dwyoon@gsnu.ac.kr 


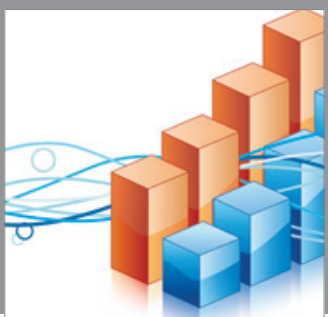

Advances in

Operations Research

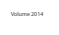

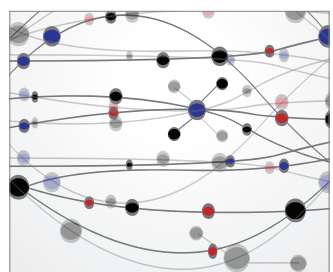

\section{The Scientific} World Journal
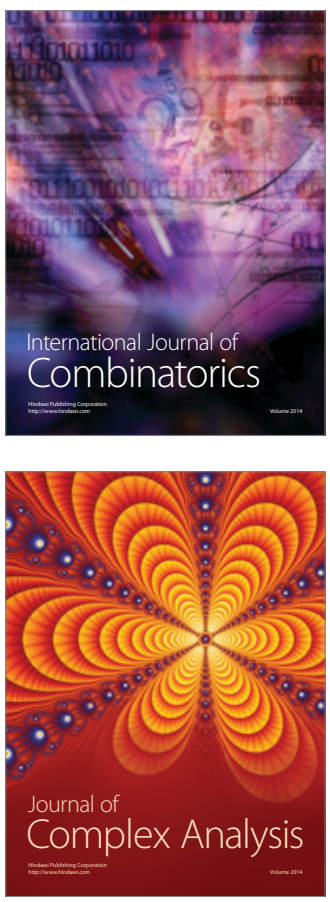

International Journal of

Mathematics and

Mathematical

Sciences
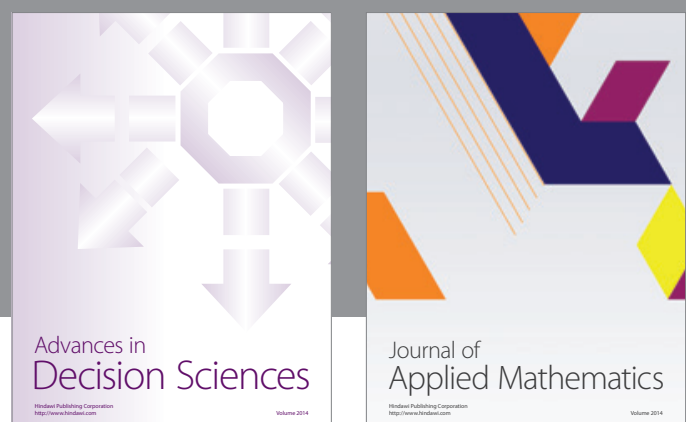

Journal of

Applied Mathematics
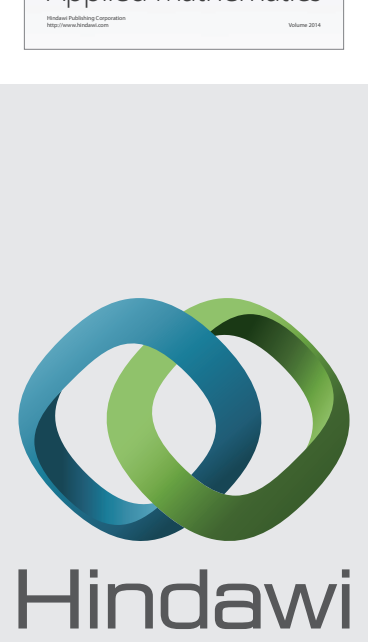

Submit your manuscripts at http://www.hindawi.com
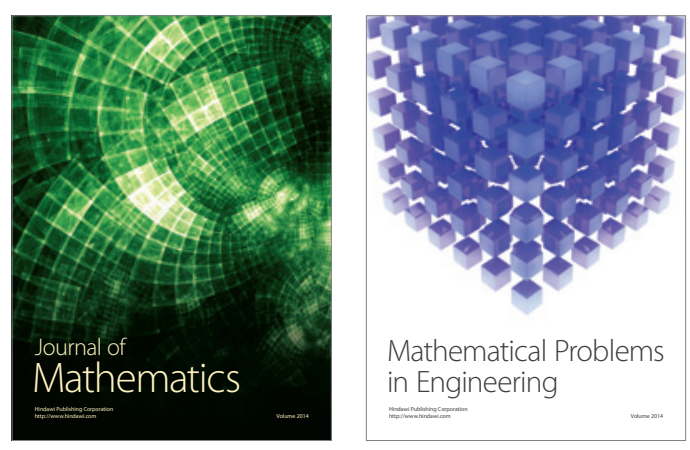

Mathematical Problems in Engineering
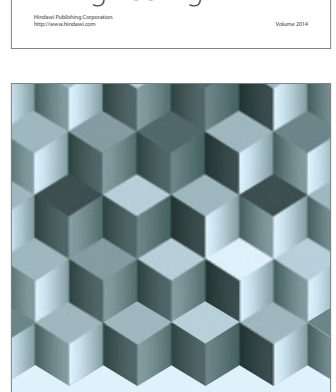

Journal of

Function Spaces
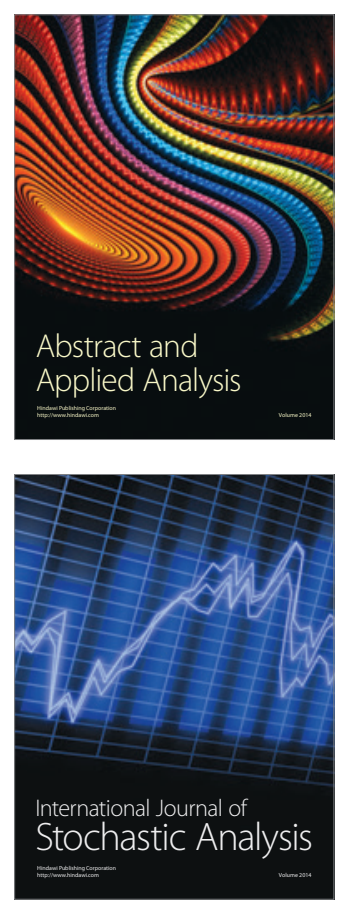

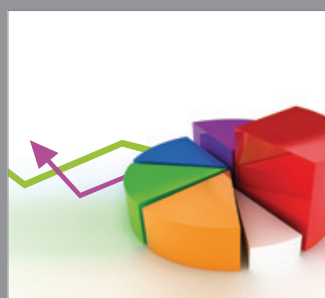

ournal of

Probability and Statistics

Promensencen
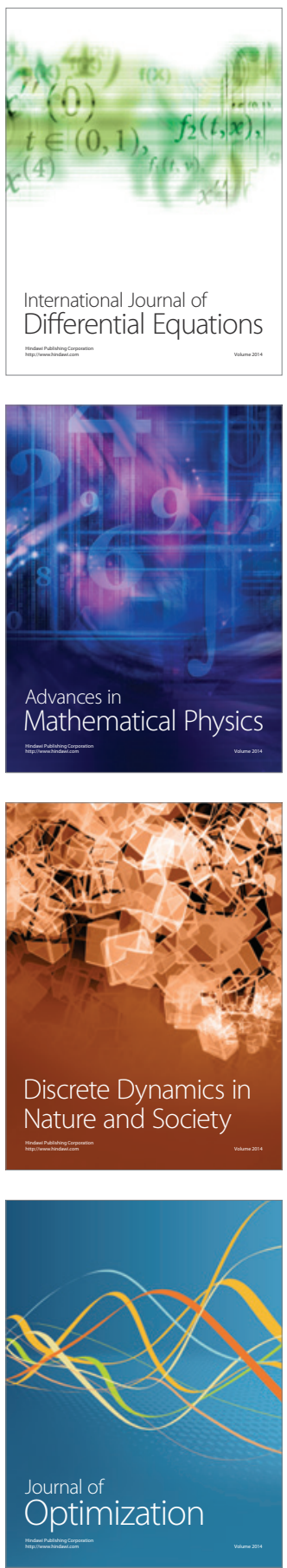